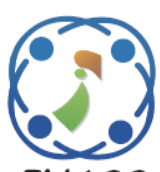

\title{
Enhanced Ant Colony Optimization for Routing in WSNs An Energy Aware Approach
}

\author{
Yasameen Sajid Razooqi ${ }^{1 *}$ \\ Muntasir Al-Asfoor ${ }^{1}$ \\ ${ }^{I}$ Computer Science Department, College of Computer Science and Information Technology, \\ Al-Qadisiyah University, Iraq \\ * Corresponding author's Email: yasameen.sajid@qu.edu.iq
}

\begin{abstract}
Routing is one of the most critical issues in Wireless Sensor Networks (WSN) as it differs from routing in classical networks. The dynamic nature of WSN environment and the constrained limited resources availability have made routing process a real challenge to industry and academia. One of the best solutions in this routing case is a heuristic search to find the best and optimal path. Thus, many heuristic optimization algorithms are used to enhance routing performance in WSNs. Ant Colony Optimization (ACO) is a well-known heuristic algorithm which used in WSNs, as it showed an admirable performance improvement routing context. In this paper, two routing protocols are proposed: modified Ant Colony Optimization for flat routing protocol (MACOF), and the second is Naïve Baye's with ranking for flat routing protocol (NBRF). We have used inverse of the distance between the node and it's neighbours in the probability equations of ACO and Naïve Baye's formulas, along with pheromone and residual energy. Those formulas have been used in choosing the candidate between neighbors for the next hop that will lead to consume less average energy of all nodes in each round. In addition, in the first protocol we have adjusted ACO's parameters values after several experiments, and in the second protocol we have proposed ranking for network training before start working. The experimental analysis has been done using varied scenarios of networks topologies. Furthermore, the experimental results have been compared and contrasted against the well-known ACO approaches and IOP routing protocols. The performance of the proposed methods of MACOF and NBRF have been shown in terms of throughput, energy consumption, network lifetime, the size of data transferred over the network, and the length of paths travelled by the packets.
\end{abstract}

Keywords: Wireless sensor networks, Routing algorithms, Ant colony optimization, Energy consumption.

\section{Introduction}

WSN consist of hundreds to thousands of very small devices called sensors for collecting data from the surrounding environment and transmitting them to a base station called Sink [1]. The sensing data varied according to the environment and the required application or purpose such as temperature, pressure, vibrations, noise, wind velocity, and humidity data. After receiving these data by the sink they can be more analysed and processed and may be sent them to remote user or cloud throughout the integration with IoT. The applications of IoT are numerous like forests and oceans monitoring, Nuclear reactor area management, health care applications, etc. [2]. Figure 1 illustrates WSN and its integration with IoT. Each sensor consists of three main components, the sensing unit for data acquisition which is the main function of the sensor, the processing unit that is contained the storage memory, and the communication unit for sending and receiving data. The sensor may also contain other components such as a location finding system (i.e. GPS) and mobilizer for mobility management (MM) in the case of the mobile sensor, both of them are expensive and consume sensor great energy [3]. Solar cells may be added as a source of energy in sensor [4]. As a power source, the sensor depends on the battery in its operation, that is the main problem in WSNs since it cannot be rechargeable or replaceable. The reason for that is the far and 


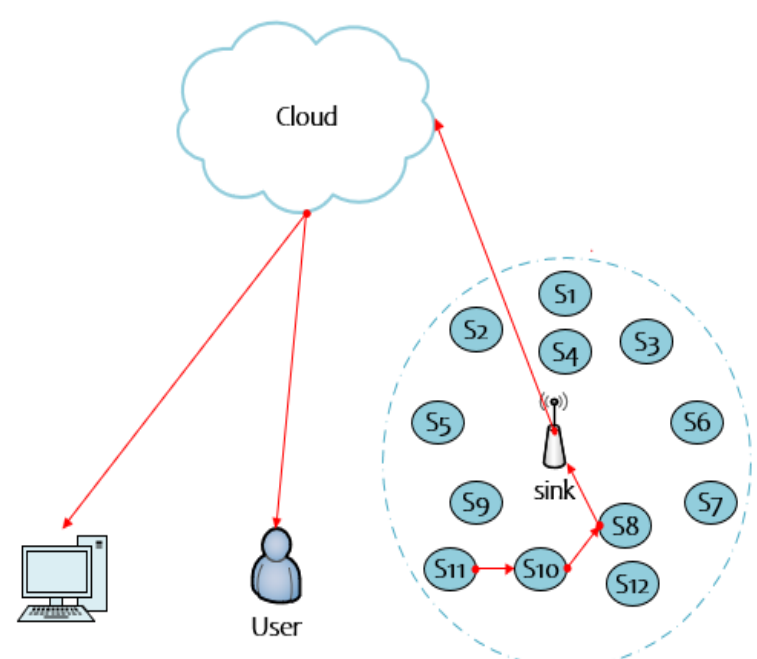

Figure. 1 Illustrates WSN components and their integration with IoT

dangerous area that sensors disseminated in, that almost cannot be reached by humans, and the low cost and small size of the sensor [5]. The greatest amount of energy is consumed in communication where it does not compare to the amount consumed in sensing and processing. For those reasons in addition to the big number of sensors that disseminated in a wide geographical area which cannot be managed manually, dynamic routing in WSN becomes the most critical issue to reduce the energy consumed in communication by reducing the distances travelled by the packets and the number of hops to reach to the destination(sink) [6]. This goal is achieved through the use of artificial intelligence techniques and distributed algorithms that can learn the network in finding the success paths with the least distance and hence minimum energy [7].

In this context, we propose two protocols for flat routing in WSN, the first is modified Ant Colony Optimization for flat routing protocol (MACOF), and the second is Naïve Baye's with ranking for flat routing protocol (NBRF). The organization of the paper will be as following: Section 2 will illustrate the motivation of this work and proposed contributions. Related work will explain in Section 3. Proposed model will describe in Section 4 in details. then experimental result and analysis and discussion part in Section 5, finally Section 6 contain conclusion and future scope.

\section{Motivation and contributions}

Routing in WSN is a very important factor that effecting on network lifetime and overall performance. As mentioned in the previous section, sensors depend on the battery as a power supply and the greater amount of energy is lost in communication, therefore managing routing to reduce the network energy consumption with high throughput at the same time. We suggest using AI algorithms in selecting the next hop or candidate neighbour to send the packet for since it is the main difference in WSN routing from other types of networks. Each sensor has partial knowledge about the network that is just about his neighbours whose within its communication range rather than the whole network. Most research about routing in WSN uses hierarchical routing that working on partitioning the network into clusters with a central cluster head in each cluster that collects data from the sensor through one hop and each cluster head sending them to the sink. The complexity in the flat protocol that we working on is in sending packets through a long multi-hop path to the sink from each sensor without using a central node, forming a fully decentralize or peer-to-peer $(\mathrm{P} 2 \mathrm{P})$ routing protocol.

We propose two smart routing protocols, in the first we used modified Ant Colony Optimization to find the next-hop node in searching for the sink (the destination). The modification was in the probability equation by adding two heuristic functions using the distance between nodes and the energy of the recipient node, in addition to the pheromone concentration. Also, we have adjusted ACO's parameters values $(\alpha, \beta, \rho, \gamma$, and $\mathrm{Q})$ after several testing results and analyses using different networks topologies to reach the suitable parameters' values for ACO to be used in flat routing protocol in WSNs. In the second protocol, we used Naïve Baye's classifier for selecting the nodes that lead to the sink after training the network with a ranking mechanism through adding scores to the nodes along the success paths.

In addition, we propose to reduce the allowable communication range of the sensor rather than using an actual one to prevent each sensor from sending packets across long distances which consume great energy according to the energy model in section 5.2.2. We also reduce the TTL value (number of hops) as possible according to the size of the network. Finally, we propose to use super sensors in the region near the sink to solve the problem of flat protocols in WSNs of fast nodes death as a consequence of high load on them.

\section{Related work}

In this section, we will show some researches that are about reducing energy consumption in WSN through hierarchical or flat routing. In [8] the authors used Capsule neural network (CNN) for developing a learning model for detecting the nodes 
that are appropriate to select them as cluster heads inside the cluster by identity records and also to select the forward nodes that are outside the clusters using shortest path selection to reduce the energy consumption in WSN. the researchers in [9] introduced techniques for clustering and routing management. They used gravitational force in addition to fuzzy rules for constructing clusters and routing to increase the lifetime of the network. Furthermore, they used a deductive inference system based on fuzzy logic for choosing suitable nodes as cluster head CH. Energy-efficient scalable clustering protocol (EESCP) in [10], takes into account distances between clusters and inside the cluster to produce equiponderant clusters. In this protocol, a Particle swarm optimization technology based on Dragonfly's algorithm (DA-PSO) was utilized to choose cluster heads. In it also, a new energyefficient fitness function was used to choose an optimal collection of $\mathrm{CHs}$, the above studies are about hierarchical routing protocol which suffering from westing time in choosing and changing cluster head during network life time in addition fast death of the cluster head as it's working on collecting sensing data from all sensors in the cluster and send them to the sink beside his work of sensing data. The researchers in [11] produced a new routing protocol called Intelligent Opportunistic Protocol (IOP) make use of artificial intelligence for choosing the probable relay node by utilizing naïve Baye's classifier. Packet Reception Ratio, Residual Energy of nodes, and Distance were the features used for getting the probability of a node to be a forwarder for next-hop. ACO is utilized in [12] to choose the next hop between neighbours using the residual energy and pheromone as the heuristics functions for calculating the probability of each node. The same algorithm is used in [13] for the same purpose of choosing the best neighbour but with a different function for the residual energy as the heuristic function in ACO.

The research work in $[12,13]$, the used heuristic function based on higher residual energy that did not granting reaching the sink in less hops which lead to decreasing throughput with high energy consumption. The main limitation of flat routing protocol, the sensors which near the sink loss their energy rapidly du to work's load in transmitting the sensing data of the other sensors. Unlike the proposed protocol the sensors near the sink depending on solar cells as a power supply and using artificial intelligence techniques to minimize the length of the success path.

\section{Proposed model}

\subsection{Network model}

There are two models for sensor distribution in the monitoring area :(i) pre-planned mode is the easier situation in which the monitoring area can be reached to deploy and manage sensors manually which provides good coverage with less number of sensors. (ii) ad-hoc mode is very significant in harsh or far environments or systems that cannot be reached, which requires more sensors deployed randomly to provide the required coverage [14]. The second model is the distribution that was followed in the network's construction phase, where $\mathrm{N}$ sensors were deployed randomly in a square area. Three networks were used with different numbers of nodes and network areas as follows: 80 nodes in 100*100 meters, 160 nodes in $200 * 200$ meters, 240 nodes in $300 * 300$ meters. Each sensor sends a hello packet to its neighbours for telling them about ID, location, and energy information. Data transfer has been classified into (i) event-driven in which data transmission starts after a certain event, (ii) timedriven sensed data transmitted to the destination in a specific time, and (iii) query-driven data transmitted after some request reached the sensor [6]. In our model, the first type was suggested to allow a specific group of sensors to send their sensing data at each round of work, to simulate that, this group of sensors was chosen randomly every round.

\subsection{Energy model}

We proposed all the sensors have equal initial energy, with time each sensor will have different energy due to the energy loss in sensing, processing, and communication. The energy consumed in sensing and processing can be ignored as it is very small compared with energy consumed in communication. Each node has a neighbours' information table like ID, residual energy, and location. The distance between nodes is calculated according to the Euclidean distance and the residual energy is updated in the table after any change in the value. The energy consumption is calculated using the equations in [15], one of the two equations is implemented depending on the distance between the sender and the receiver sensors. If the distance is less than the threshold $d 0$, then free space model is implemented, else the multipath fading model is implemented to calculate the energy of sending a packet of size $l$ bit: 


$$
E_{T}(l, d)=\left\{\begin{array}{cc}
l E_{\text {elec }}+\varepsilon_{f s} d^{2} & \text { for } d<d_{o} \\
l E_{\text {elec }}+\varepsilon_{m p} d^{4} & \text { for } d \geq d_{o}
\end{array}\right.
$$

$E_{\text {elec }}$ is the energy required by the electronic circuit, while $\varepsilon_{f s}$ and $\varepsilon_{m p}$ are the energy consumed for an amplifier in free space and multipath fading channels.

In the receiver sensor, energy lost in receiving $l$ bits is:

$$
E R(l)=l E_{\text {elec }}
$$

\subsection{Ant colony optimization for flat routing protocol (MACOF)}

ACO is a robust and effective heuristic search algorithm, the ACO probability of searching paths depends on former search results [16]. ACO was invented by Dorigo et al. in the 1990s, as an imitation of ants' searching for food in nature [17]. Ants in real-life work with each other for finding the right direction of food by left a chemical called "pheromone" along the path they follow to contact one another [18]. At the beginning of network operation, all paths have an equal probability for the ants to choose among them since there is no pheromone. After finding the food a backward ant is sent for putting the pheromone along the path from the food location to the source [19]. Over times, pheromone concentration reduces due to evaporation, and the right path to food will gain high pheromone concentration thus will be followed by other ants because the ant follows the path with the highest pheromone and the bad paths will be neglected [20].

We suggested using ACO in WSN to be performed by sensors since it is a distributed algorithm [21]. We propose to utilize the information in the neighbour's table such as distance and the residual energy in the ACO probability equation along with pheromone value, which is also will be added to the table, to choose the next-hop neighbour and subsequently the best path to the destination, aiming to reduce the energy consumption with high throughput [22]. The ACO probability equation in [23] is as follow:

$$
\begin{aligned}
& p_{i j}^{m}(t) \\
& =\left\{\frac{\left[\tau_{i j}(t)\right]^{\alpha} \cdot\left[n_{i j}(t)\right]^{\beta}}{\sum v_{m} \in v_{\text {allowed }}^{m}\left[\tau_{\text {im }}(t)\right]^{\alpha} \cdot} \begin{array}{l}
{\left[n_{\text {im }}(t)\right]^{\beta}} \\
\text { if } v_{j} v_{\text {allowed }}^{m}
\end{array}\right.
\end{aligned}
$$

Where, pijm is the probability of ant $\mathrm{m}$ or packet to transfer from node $\mathrm{i}$ to node $\mathrm{j}$ at a time or iteration $\mathrm{t}$, $\mathrm{V}$ neighbours of node $\mathrm{i}, \tau \mathrm{ij}$ is the pheromone concentration on the edge between node $i$ and node $j$, $\eta \mathrm{ij}$ is the heuristic function for a packet to move from node $i$ to node $j$, and $\alpha$ and $\beta$ are the weights of $\tau \mathrm{ij}$ and $\eta \mathrm{ij}$.

First, assume all connections between sensors have equal initial pheromone thus sensors will choose the next-hop without being affected by the pheromone value, then the pheromone value will be updated with each round. We propose to use two heuristics functions besides the pheromone value by making use of the distance and the residual energy and with an update to the values of the parameters $\alpha$, $\beta$ and $\gamma$

$$
\begin{aligned}
& p_{i j}^{m}(t)= \\
& \left\{\frac{\left[\tau_{i j}(t)\right]^{\alpha} \cdot\left[n_{i j}(t)\right]^{\beta} \cdot\left[\delta_{j}(t)\right]^{\gamma}}{\sum v_{m} \in v_{\text {allowed }}^{m}\left[\tau_{\text {im }}(t)\right]^{\alpha} \cdot\left[n_{\text {im }}(t)\right]^{\beta} \cdot\left[\delta_{j}(t)\right]^{\gamma}}\right. \\
& \text { if } v_{j} \quad v_{\text {allowed }}^{m}
\end{aligned}
$$

\section{Where,}

$\eta \mathrm{ij}$ is the inverse of the distance (1/d) between nodes $\mathrm{i}$ and $\mathrm{j}$, and $\delta \mathrm{j}$ is (initial energy - residual energy of $j$ ).

The pheromone values will be changed in all connections in the network for two reasons, the first reason is the pheromone evaporation in all edges in the network, and the second is the pheromone concentration increasing along the success paths. To perform the pheromone updates we used the equations in [24] but with some changes as follow:

$$
\tau_{i j}(t+\Delta t)=(1-\rho) \cdot \tau_{i j}(t) \text { for all edge }
$$

And for all success paths use Eq. (6).

$$
\tau_{i j}(t+\Delta t)=\tau_{i j}(t)+\Delta \tau_{i j}(t)
$$

Where $\Delta \tau_{i j}(t)=\sum_{m=1}^{M} \Delta \tau_{i j}^{m}(t)$,

And $\Delta \tau_{i j}^{m}(t)=\left\{\begin{aligned} \frac{1}{L_{m}}, & v_{i}, v_{j} \in P \operatorname{Path}_{m} \\ 0, & \text { otherwise }\end{aligned}\right.$

\section{Where,}

$\rho$ is the pheromone evaporation degree (between 1 and 0$), \Delta \tau i j m(t)$ is the amount of pheromone left by ant, and Lm is the length of the success path.

After transmitting sensed data several times, the sensors will learn to select the best neighbour because of the pheromone value raising in the success paths and minimized in other edges those who did not lead to the destination. And hence, the 
success of packet delivery will increase and energy lost will be minimized. The steps of this protocol are shown in Algorithm 1.

Algorithm 1. Ant colony optimization for flat routing protocol (MACOF)

\begin{tabular}{ll}
\hline Algorithm 1: ACO routing algorithm \\
\hline Result & Success path=" all success path" \\
\hline Initialization & \\
& Visited node =" false" \\
& Success path=" “ \\
& Energy node=5 J \\
& Set no. of. hops \\
& Set $\mathrm{d}_{\mathrm{o}}$, Set $\mathrm{E}_{\text {elec }}$, Set $\mathrm{E}_{f s}$, Set $\mathrm{E}_{m p}$ \\
& Set $\mathrm{L}=$ packet size \\
& Source $=\mathrm{G}$. node \\
\hline
\end{tabular}

Steps

Received energy $=\mathrm{L}^{*} \mathrm{E}_{\text {elec }}$

1. While no. of. hops! $=0$ do

2. Sort (source. neighbours) based on maximum ACO probability using Eq. 4

3. for $\mathrm{i}=1$ to no . of. neighbours do

4. node $=$ select source. neighbours[i]

5. $\quad$ if node. visited $=$ false then

6. $\quad$ node. visited $=$ true

7. if distance (source. node) $<\mathrm{d}_{\mathrm{o}}$ then

8. $\quad$ transfer. energy $=\left(l \mathrm{E}_{\text {elec }}+\mathrm{E}_{f s} d^{2}\right)$

9. Else

10 transfer. energy $=\left(l \mathrm{E}_{\text {elec }}+\mathrm{E}_{m p} d^{4}\right)$

$11 \quad$ End

12 if source. energy > transfer. energy

then

13 transfer. energy

14 if node. energy > received. energy

then

15 received. energy node. energy $=$ node. energy -

16 end

end

source $=\mathrm{G}$. visited. node

no. of. hops= no. of. hops-1

update success path

end

End

if all source. neighbors=true and

source. neighbors. energy < transfer.

energy then

source $=$ pervious source

no. of. hops $=$ no. of. hops -1

Go to step 3

else

if source is sink then

return (success path)

update (success path, nodes pheromone according Eq 6

break

else

if no. of. hops $==0$ then return (fail)

else

Go to step 2

end

end

End

40 update (all nodes. evaporations based on Eq 5)

41 End

Algorithm 2. Network training with assigning ranks Algorithm 2: Learning based on Ranking Concept

\begin{tabular}{ll}
\hline Result & Success path=" all success path" \\
\hline Initialization & \\
& Visited node =" false" \\
& Success path=" " \\
& Energy node=5 J \\
& Set no. of. hops \\
& Set $\mathrm{d}_{\mathrm{o}}$, Set $\mathrm{E}_{\text {elec }}$, Set $\mathrm{E}_{f s}$, Set $\mathrm{E}_{m p}$ \\
& Set $\mathrm{L}=$ packet size \\
& Source $=\mathrm{G}$. node \\
\hline
\end{tabular}

Steps

1. While loop ! =N do

2. Set source $=\mathrm{G}[$ loop $]$

3. for $\mathrm{i}=1$ to no . of. hops do

4. node $=$ select source. neighbors[randomly]

5. if node. visited $=$ false then

6. $\quad$ node. visited $=$ true

7. if distance (source. node) $<\mathrm{d}_{0}$ then

8. $\quad$ transfer. energy $=\left(l \mathrm{E}_{\text {elec }}+\mathrm{E}_{f s} d^{2}\right)$

9. else

10

11

12

13

14

15

16

17

18

19

20

21

22

23 if all source. neighbors=true and source. neighbors. energy < transfer. energy then 


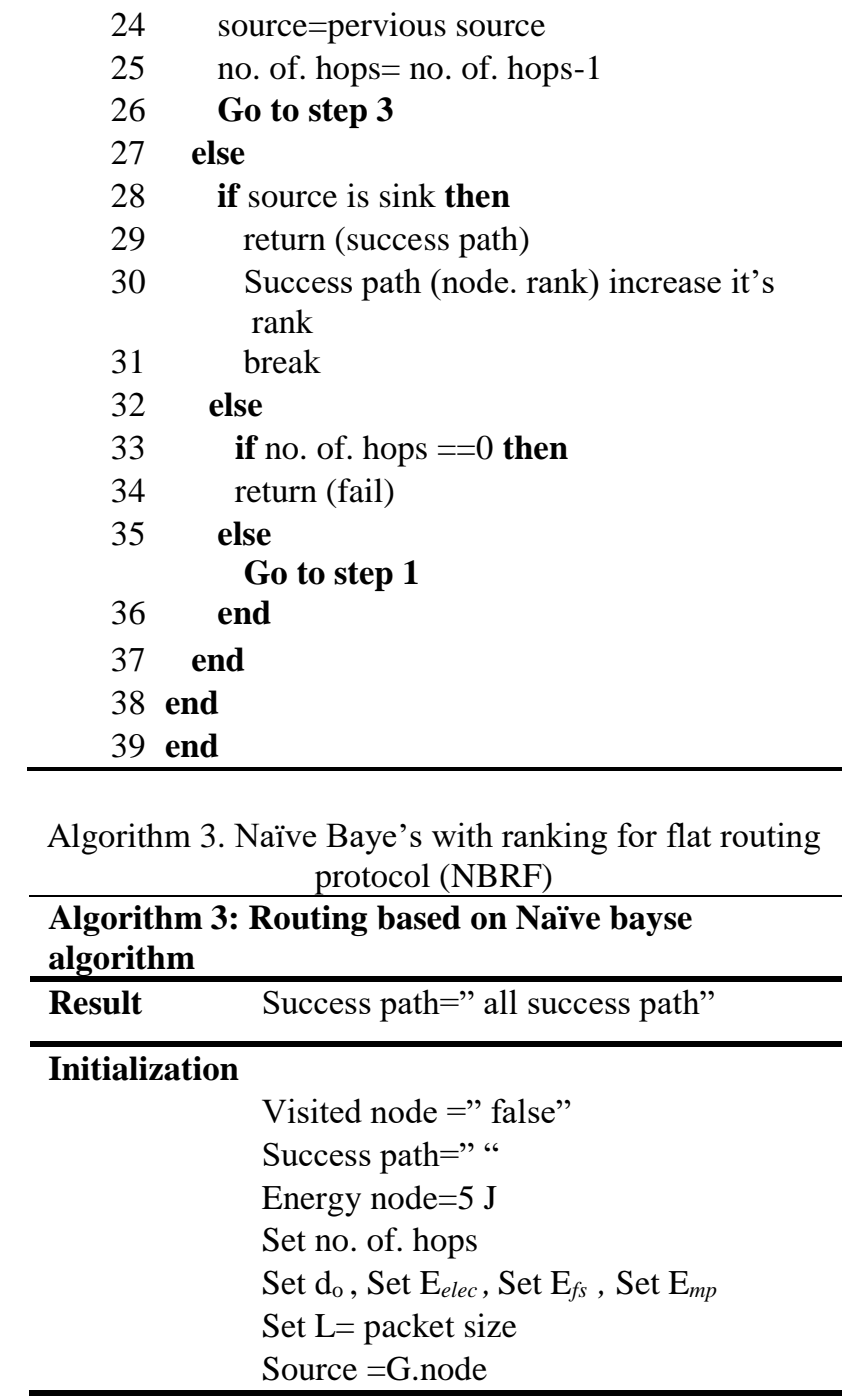

1. Received energy $=\mathrm{L} * \mathrm{E}_{\text {elec }}$

2. While no. of. hops! $=0$ do

3. Sort (source. neighbors) based on maximum ACO probability using Eq. 4

4. for $i=1$ to no . of. neighbors do

5. node $=$ select source. neighbors $[\mathrm{i}]$

6. $\quad$ if node. visited $=$ false then

7. $\quad$ node. visited $=$ true

8. if distance (source. node) $<\mathrm{d}_{0}$ then

9. $\quad$ transfer. energy $=\left(l \mathrm{E}_{\text {elec }}+\mathrm{E}_{f s} d^{2}\right)$

10 else

11 transfer. energy $=\left(l \mathrm{E}_{\text {elec }}+\mathrm{E}_{m p} d^{4}\right)$

12 end

13 if source. energy > transfer. energy then

14 source. energy= source. energytransfer. energy

15 if node. energy > received. energy then

16 node. energy= node. energyreceived. energy

17

18

$$
\text { end }
$$

end
19

20

21

22

23

\subsection{Naïve Baye's with ranking for flat routing protocol (NBRF)}

In the second protocol, we proposed using Naïve Baye's classifier with the ranking concept in the networks to build a machine learning model for routing in WSN. The ranking is sorting network objects according to some order such as importance, popularity, similarity, etc., it is used in networks by assigning scores to nodes, objects, or pages [25]. NBRF consist of two phases, in the first phase we employ the ranking concept in routing by adding ranks or scores to the nodes, initially equal to 1 for every node. Then, when the network starts in operation, each sensor sends a hello packet to one of its neighbours which in turn do the same, this will be repeated until reaches the sink or access the TTL limit. The sensors that led to the sink or the nodes along the success paths will be assigned additional scores as feedback from the sink to those sensors $($ rank $=$ previous rank $+\Delta$ rank $)$, this scenario of learning is applied only at the first time of sensors work as shown in Algorithm 2, then the sensors start to send the sensing data to the sink through multihop paths, as a second phase. In this stage, we suggest using Naïve Baye's equations for probability as shown in equation no.7 to calculate the likelihood of a sensor S sending the packet to one of its neighbours N. After calculating the probability for each neighbour, the source node will 
choose the neighbour who has the maximum probability for sending the packet, as shown in Algorithm 3.

The features we used to apply the Naïve Baye's probability are ranks of neighbours $\mathrm{X} 1$, the inverse of the distance $(1 / \mathrm{d})$ between the node and its neighbours $\mathrm{X} 2$, and the residual energy of the neighbours (initial energy - residual energy of $\mathrm{j}$ ) X3.

$$
\begin{aligned}
(N \mid S)= & P\left(X_{1} \mid N\right) * P\left(X_{2} \mid N\right) * P\left(X_{3} \mid N\right) \\
& P\left(N \mid X_{i}\right)=\frac{P\left(X_{i} \mid N\right) * P(N)}{P\left(X_{i}\right)} \\
\text { and hence } \quad & P\left(X_{i} \mid N\right)=\frac{P\left(N \mid X_{i}\right) * P\left(X_{i}\right)}{P(N)}
\end{aligned}
$$

\section{Experimental result and analysis}

In this section, we present the networks attributes, simulation environment, and parameters with the performance evaluation result of the two suggested protocols according to the performance metrics we had used. Also, we will introduce the results of the two protocols comparing with the IOP protocol [11] and ACO routing protocol [12] of flat routing in WSNs.

\subsection{Simulation environment}

For simulating network configuration, routing protocols, and analysing results we used Python language with Network $X$ package. To proof the performance of our protocols we have used three different networks with different features $(80$ nodes in $100 * 100$ meters, 160 nodes in $200 * 200$ meters, 240 nodes in $300 * 300$ meters) as mentioned in section 4.1. Parameters that are used in the network model, energy model, and the ACO will be described in table 1 . This parameter will used in all our experimental analysis for the four protocols.

Table 1. Experimental parameters

\begin{tabular}{|l|l|}
\hline Parameters & Value \\
\hline $\begin{array}{l}\text { The initial energy of the } \\
\text { sensor }\end{array}$ & $5 \mathrm{~J}$ \\
\hline Number of sinks & 1 \\
\hline Location of sink & Centre of area \\
\hline Packet size & 1024 \\
\hline$E_{\text {elec }}$ & $50 \mathrm{~nJ} / \mathrm{bit}$ \\
\hline$\varepsilon_{m p}$ & $0.0013 \mathrm{pJ} / \mathrm{bit} / \mathrm{m} 4$ \\
\hline$\varepsilon_{f s}$ & $10 \mathrm{pJ} / \mathrm{bit} / \mathrm{m} 2$ \\
\hline$d_{0}$ & 50 \\
\hline$\alpha$ & 2 \\
\hline$\beta$ & 3 \\
\hline$\gamma$ & 2 \\
\hline Initial pheromone & 1 \\
\hline$\rho$ & $0.5-1$ \\
\hline$Q$ & 1 \\
\hline
\end{tabular}

\subsection{Performance metrics}

We have evaluated MACOF and NBRF routing protocols based on aspect throughput, energy consumption, network lifetime, the size of data transferred over the network, and the length of paths travelled by the packets. And compare them with Intelligent Opportunistic routing protocol (IOP) under the same simulation networks and parameters to prove the efficiency of the algorithms in the mentioned metrics.

\subsection{Result}

In the following section, we will show each one of the aforementioned metrics calculated for the two routing protocols MACOF and NBRF compared with the IOP routing protocol and ACO-based routing according to iterations or rounds of network operation. The results will consist of the experiments of the protocols on the three networks we had mentioned $(80,160$, and 240 nodes) using the parameters shown in Table 1. Succuss ratio will be shown to explain the network throughput which measures how many packets arrive at the sink successfully.

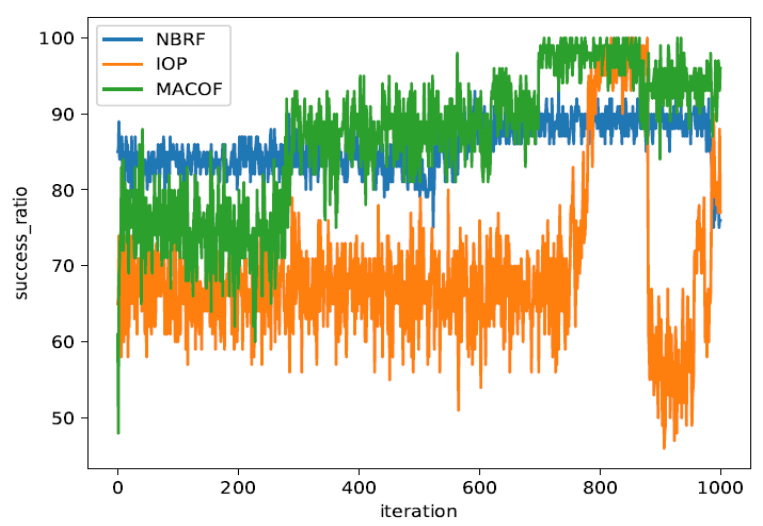

Figure. 2 Success ratio in 240 nodes ( $300 * 300 \mathrm{~m}^{2}$ ) MACOF and NBRF compared with IOP

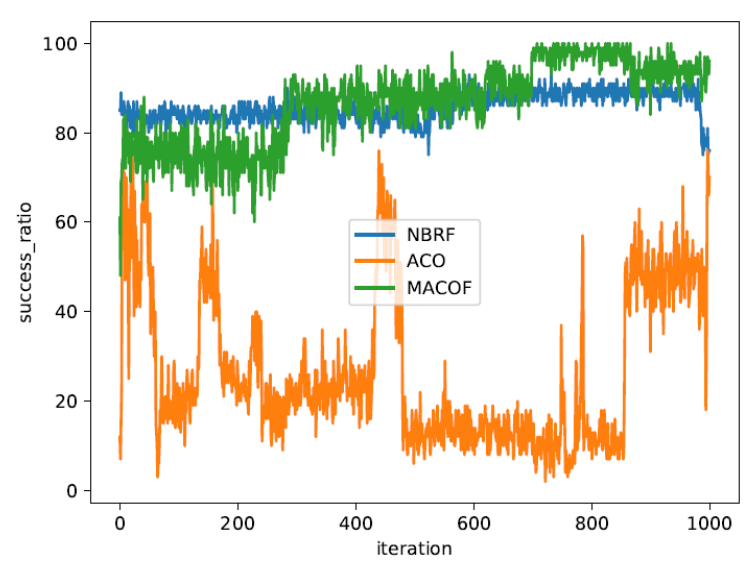

Figure. 3 Success ratio in 240 nodes $\left(300 * 300 \mathrm{~m}^{2}\right)$ MACOF and NBRF compared with ACO 
As shown above in Fig. 2, success ratio of the proposed model: MACOF and NBRF routing protocols, achieved good result compared with IOP. While Fig. 3 for same proposed models compared with ACO based routing protocol, also achieved good result than ACO.

Energy consumption is the second metric of the network performance that refers to the overall energy expended in sending and receiving packets in each round according to the energy model in Section 4.2. Fig. 4 to 7 show the energy consumption of three topologies, each one shows the comparison of the proposed protocols with IOP routing protocol and ACO. In all the below figures the proposed models give good result unlike IOP and ACO in saving energy.

Network lifetime is the time from the beginning of network operation until the first node exhausting its energy. It is an important metric in networks because of the degradation in network performance after nodes start dying.

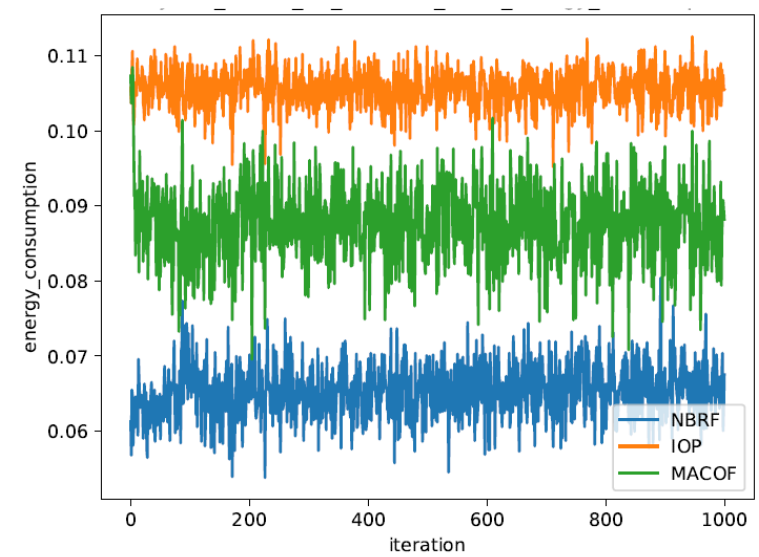

Figure. 4 Energy consumption in 80 nodes $\left(100 * 100 \mathrm{~m}^{2}\right)$ MACOF and NBRF compared with

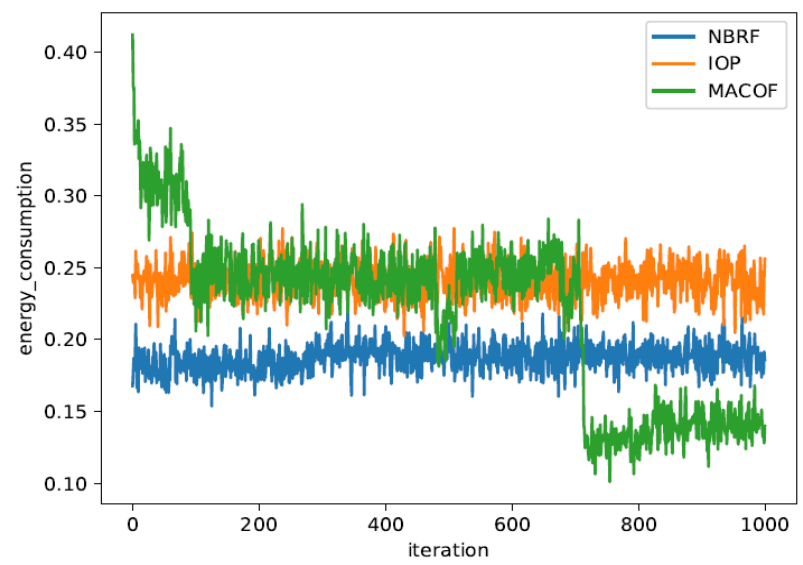

Figure. 5 Energy consumption in 160 nodes $(200 * 200$ $\mathrm{m}^{2}$ ) MACOF and NBRF compared with IOP

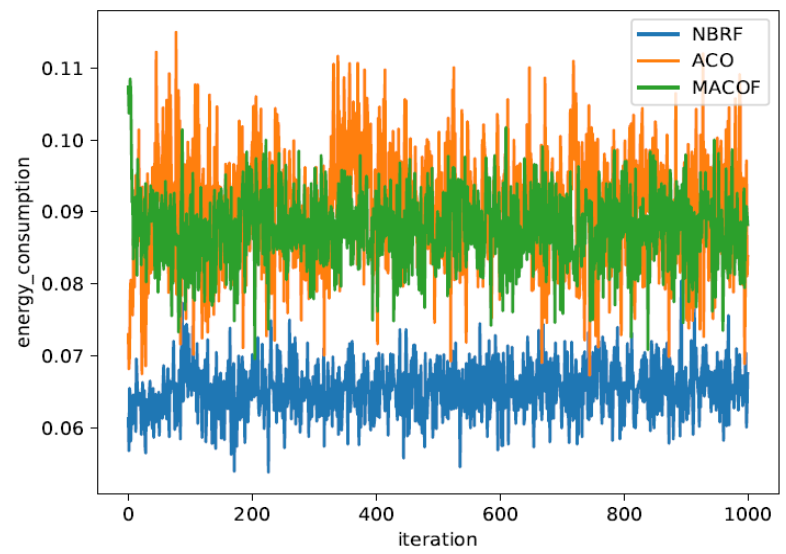

Figure. 6 Energy consumption in 80 nodes $\left(100 * 100 \mathrm{~m}^{2}\right)$ MACOF and NBRF compared with ACO

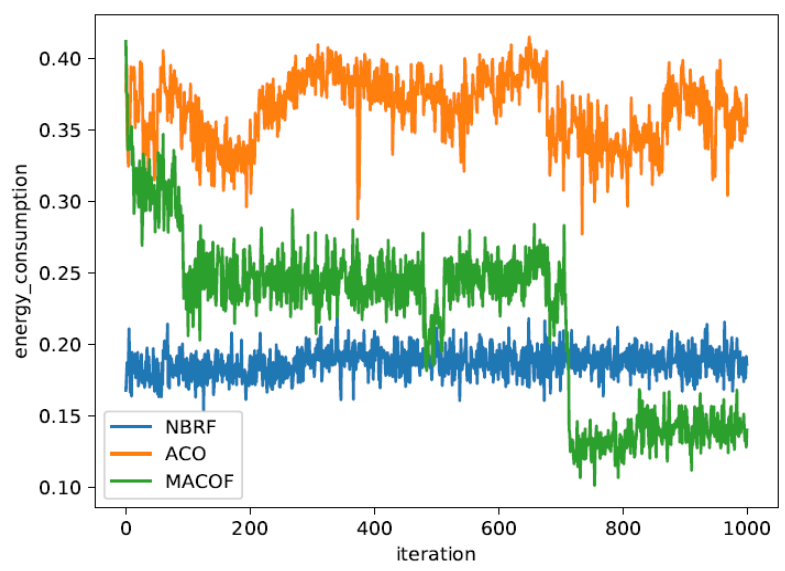

Figure. 7 Energy consumption in 160 nodes ( $200 * 200$ $\mathrm{m}^{2}$ ) MACOF and NBRF compared with ACO

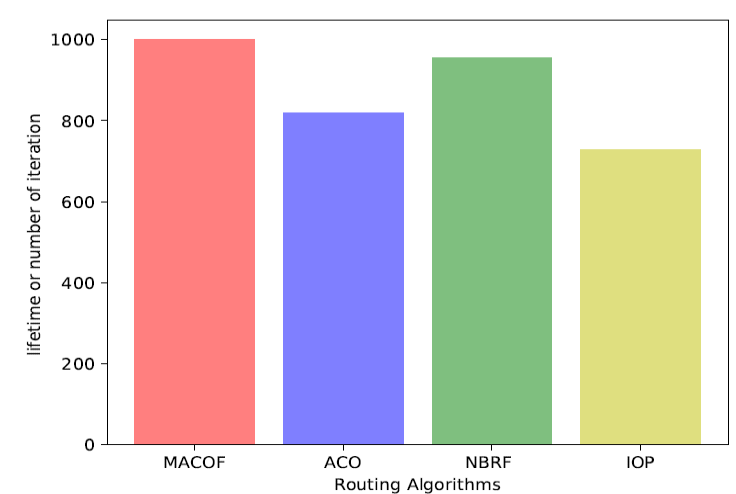

Figure. 8 Lifetime in 240 nodes $\left(300 * 300 \mathrm{~m}^{2}\right.$ ), MACOF and NBRF compared with ACO and IOP

Fig. 8 shows network life time of four protocols in which MACOF and NBRF eclipsed on ACO and IOP.

Finally, two metrics will be shown to prove the reason behind the reduction in energy consumption: the total size of data transferred over the network in each round of network operation, measured in a bit, and the second is the total length of paths travelled by the packets over the network in each round, 
measured in meter. Those two metrics explain the excellence of the two proposed protocols in energy consumption that's because distance and data size have a direct effect on energy used in sending and receiving packets. Fig. 9 to 12 show shorter distances travelled by packet in our proposed protocols compared with IOP and ACO. In addition, the data size travelled over WSNs was less than other compared protocols.

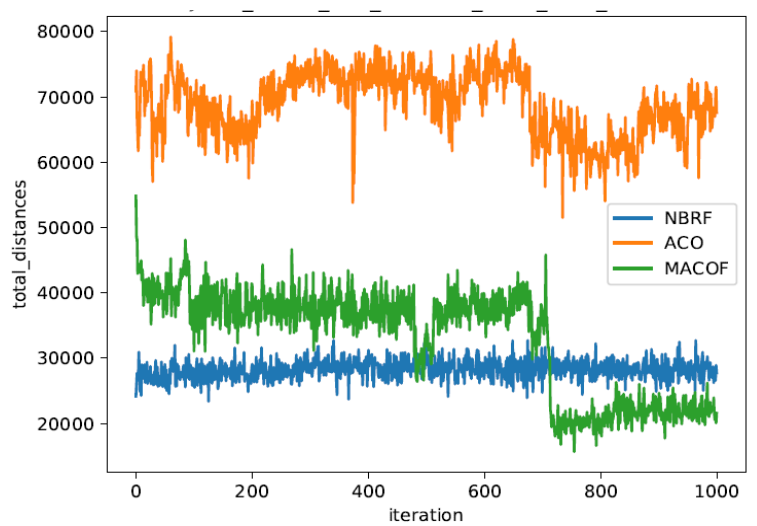

Figure. 9 Total distances in 160 nodes $\left(200 * 200 \mathrm{~m}^{2}\right)$ MACOF and NBRF compared with ACO

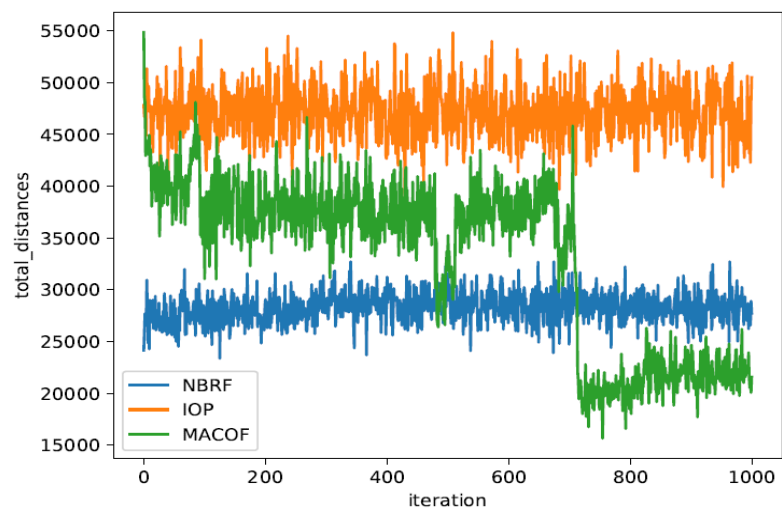

Figure. 10 Total distance in 160 nodes $\left(200 * 200 \mathrm{~m}^{2}\right)$ MACOF and NBRF compared with IOP

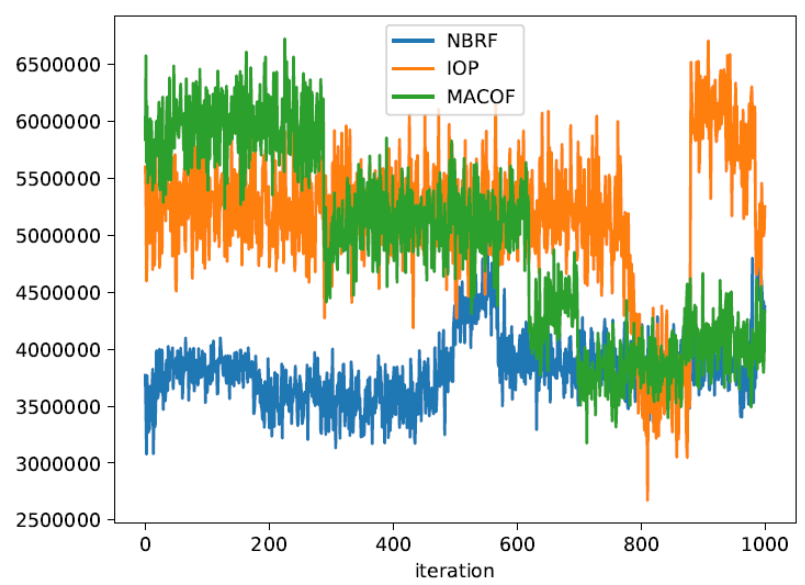

Figure. 11 Total data size in 240 nodes $\left(300 * 300 \mathrm{~m}^{2}\right)$ MACOF and NBRF compared with IOP

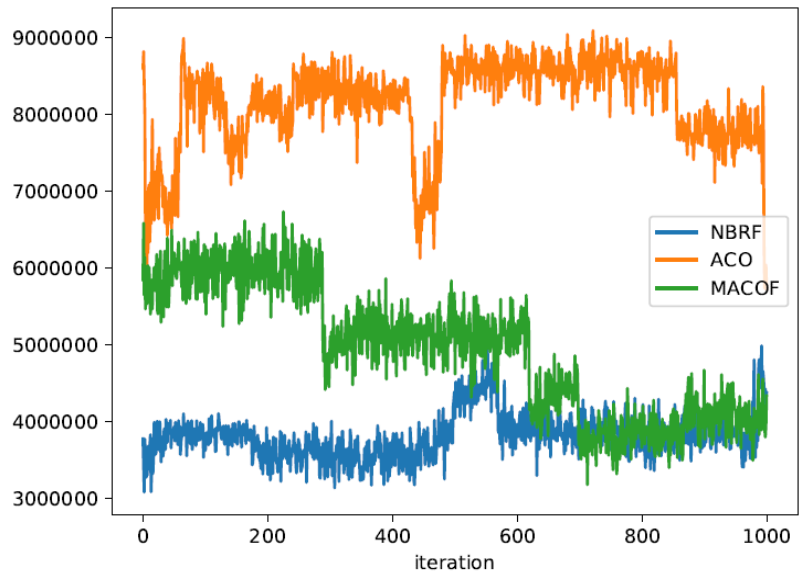

Figure. 12 Total distances in 240 nodes $\left(300 * 300 \mathrm{~m}^{2}\right.$ ) MACOF and NBRF compared with ACO

\subsection{Analysis and discussion}

The results in the previous section explained the efficiency of the proposed protocols in the throughput, energy consumption, and network lifetime. In MACOF routing protocol the modification in parameters $(\alpha, \beta, \rho, \gamma$, and $\mathrm{Q})$ and the choosing of the heuristic functions $(1 / \mathrm{d}$, initial energy - residual energy), make an excellent change in ACO performance for routing. The ACO depends on the residual energy of neighbours as a heuristic function that indicates choosing the node with higher residual energy. While NBRF training the network before sending the sensing data through adding ranks to the success paths affected on throughput and energy consumption comparing with IOP since it is also used Naïve Baye's in choosing candidate neighbour but with different heuristic functions. In NBRF we used (ranks, $1 / \mathrm{d}$, initial energy - residual energy) in calculating Naïve Baye's probability while in IOP they used (Packet Reception Ratio, residual Energy, and distance). NBRF is a pre-trained method obverse MACOF which is learning the best path during sending the sensing data through updating the pheromone concentration, which makes the NBRF achieve good percentages from the beginning of network operation while MACOF takes time and many rounds to reach the same percentages. On the other side, MACOF can perform well in case of network topology changes since it can update the pheromone value in each round according to the success stats in the previous round. Network topology changes may happen as a result of nodes death, node's location changes, or connections adaptation [26]. Whereas NBRF cannot be adapting ranks during network operation, the training in it happens only before transferring sensing data. 


\section{Conclusion and future work}

In this paper, two intelligent routing protocols are proposed to enhance the WSNs network lifetime by optimizing the energy consumption. The first is Modified Ant Colony Optimization for flat routing protocol (MACOF), and the second is Naïve Baye's with ranking for flat routing protocol (NBRF). In the first protocol we used modified Ant Colony Optimization by using three heuristic functions to the probability equation to find the next-hop node in searching for the sink, in addition, modify the pheromone concentration equations. Also, we have adjusted ACO's parameters values ( $\alpha, \beta, \rho, \gamma$, and $Q)$ after several testing results and analyses using different networks topologies to reach the suitable parameters' values for $\mathrm{ACO}$ to be suitable in flat routing protocol in WSNs. In the second protocol, we have Naïve Baye's classifier to select the best neighbour after training the network with ranking concept by adding scores to the nodes that lead to the sink. The proposed models were compared with two different routing methods: Intelligent Opportunistic routing Protocol (IOP) and ACO-based routing, to select the next-hop among a sensor's neighbours. The proposed algorithm was studied and analysed on three different network scenarios with the same network and energy parameters. Experimental results have shown the effectiveness of the suggested modified Ant Colony Optimization for flat routing protocol (MACOF), and Naïve Baye's with ranking for flat routing protocol (NBRF) in throughput and energy consumption, unlike ACO and IOP. In future work, we plan to work on light machine learning and real-time training for adapting the connections in the network based on residual energy and rank values to reach the sink.

\section{Conflict of interest}

The authors declare no conflict of interest.

\section{Author Contributions}

Conceptualization, methodology, validation, review and editing and supervision by $\mathrm{M} \mathrm{Al}$-Asfoor, implementation, writing - original draft preparation, validation by Yasameen Sajid.

\section{Acknowledgments}

We would like to thank Computer Science community for their support.

\section{References}

[1] M. M. Kumar and A. Chaparala, "OBC-WOA: opposition-based chaotic whale optimization algorithm for energy efficient clustering in wireless sensor network", Intelligence, 2019.

[2] K. Bajaj, B. Sharma, and R. Singh, "Integration of WSN with iot applications: A vision, architecture, and future challenges", EAI/Springer Innovations in Communication and Computing, 2020.

[3] T. K. Jain, D. S. Saini, and S. V. Bhooshan, "Lifetime optimization of a multiple sink wireless sensor network through energy balancing", J. Sensors, Vol. 2015, 2015.

[4] V. Sharma and A. Pughat, "Introduction to energy-efficient wireless sensor networks", Energy-Efficient Wireless Sensor Networks, 2017.

[5] B. Rashid and M. H. Rehmani, "Applications of wireless sensor networks for urban areas: A survey", Journal of Network and Computer Applications, Vol. 60, 2016.

[6] R. E. Mohamed, A. I. Saleh, M. Abdelrazzak, and A. S. Samra, "Survey on Wireless Sensor Network Applications and Energy Efficient Routing Protocols", Wireless Personal Communications, Vol. 101, No. 2. 2018.

[7] M. Elshrkawey, S. M. Elsherif, and M. E. Wahed, "An Enhancement Approach for Reducing the Energy Consumption in Wireless Sensor Networks", J. King Saud Univ. Comput. Inf. Sci., Vol. 30, No. 2, 2018.

[8] S. Govindaraj and S. N. Deepa, "Network Energy Optimization of IOTs in Wireless Sensor Networks Using Capsule Neural Network Learning Model", Wirel. Pers. Commun., Vol. 115, No. 3, 2020.

[9] M. Selvi, S. V. N. S. Kumar, S. Ganapathy, A. Ayyanar, H. K. Nehemiah, and A. Kannan, "An Energy Efficient Clustered Gravitational and Fuzzy Based Routing Algorithm in WSNs", Wirel. Pers. Commun., Vol. 116, No. 1, 2021.

[10] H. Singh and D. Singh, "An Energy Efficient Scalable Clustering Protocol for Dynamic Wireless Sensor Networks", Wirel. Pers. Commun., Vol. 109, No. 4, 2019.

[11] D. K. Bangotra, Y. Singh, A. Selwal, N. Kumar, P. K. Singh, and W. C. Hong, "An intelligent opportunistic routing algorithm for wireless sensor networks and its application towards ehealthcare", Sensors (Switzerland), Vol. 20, No. $14,2020$.

[12] A. E. Ghazi, B. Ahiod, and A. Ouaarab, "Improved ant colony optimization routing protocol for wireless sensor networks", Lecture Notes in Computer Science (including subseries Lecture Notes in Artificial Intelligence and 
Lecture Notes in Bioinformatics), 2014.

[13] J. Zhang, "Real-time detection of energy consumption of IoT network nodes based on artificial intelligence", Comput. Commun., Vol. 153, 2020.

[14] Z. A. Aghbari, A. M. Khedr, W. Osamy, I. Arif, and D. P. Agrawal, "Routing in Wireless Sensor Networks Using Optimization Techniques: A Survey", Wirel. Pers. Commun., Vol. 111, No. 4, 2020.

[15] S. Yousefi, F. Derakhshan, H. Karimipour, and H. S. Aghdasi, "An efficient route planning model for mobile agents on the internet of things using Markov decision process", Ad Hoc Networks, Vol. 98, 2020.

[16] K. C. Hu, C. W. Tsai, M. C. Chiang, and C. S. Yang, "A Multiple Pheromone Table Based Ant Colony Optimization for Clustering", Math. Probl. Eng., Vol. 2015, 2015.

[17] P. Li and H. Zhu, "Parameter Selection for Ant Colony Algorithm", Hindawi Publ. Corp. Math. Probl. Eng., Vol. 2016, pp. 1-12, 2016.

[18] L. V. Liping, "An improved ant colony algorithm in wireless sensor network routing", Int. J. Online Eng., Vol. 13, No. 5, pp. 174-187, 2017.

[19] A. E. Ghazi, B. Ahiod, and M. Abbad, "TLBObased routing approach for wireless sensor networks", International Journal of Intelligent Engineering and Systems, Vol. 11, No. 1, 2018.

[20] P. Huang, F. Lin, and J. Zhou, "ACO-based routing algorithm for cognitive radio networks", Math. Probl. Eng., Vol. 2013, No. 2, 2013.

[21] M. A. Asfoor and M. Fasli, "Distributed resource search in self-organising networks", Lecture Notes in Computer Science (including subseries Lecture Notes in Artificial Intelligence and Lecture Notes in Bioinformatics), Vol. 8062, 2013.

[22] H. J. A. Nasir, K. R. K. Mahamud, and E. Kamioka, "Ant Colony Optimization approaches in wireless sensor network: Performance evaluation", Journal of Computer Science, Vol. 13, No. 6, 2017.

[23] T. Zhi and Z. Hui, "An improved ant colony routing algorithm for WSNs", J. Sensors, Vol. 2015, 2015.

[24] P. Li and H. Zhu, "Parameter Selection for Ant Colony Algorithm Based on Bacterial Foraging Algorithm", Math. Probl. Eng., Vol. 2016, 2016.

[25] Y. Sun and J. Han, "Ranking Methods for Networks", Encyclopedia of Social Network Analysis and Mining, 2018.

[26] M. A. Asfoor and M. H. Abed, "The Effect of the Topology Adaptation on Search Performance in Overlay Network. In I. Jeena Jacob, F. M. Gonzalez-Longatt, S. Kolandapalayam Shanmugam, \& I. Izonin (Eds.)", Expert Clouds and Applications, pp. 65-73, 2022. 